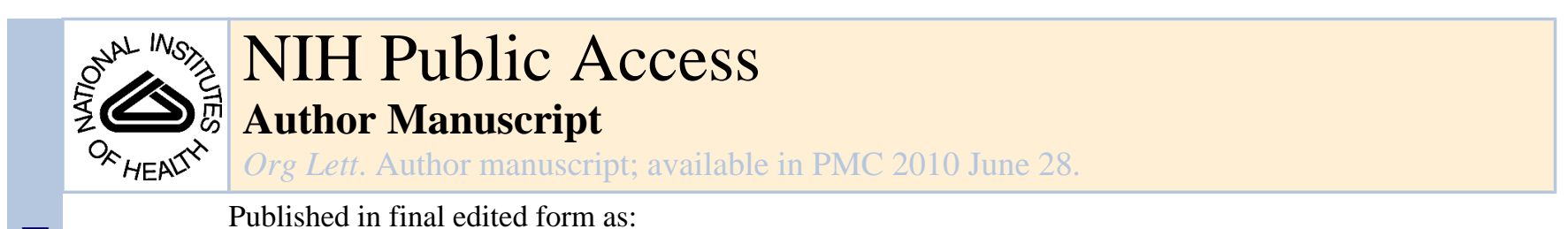

Published in final edited form as:

Org Lett. 2005 September 1; 7(18): 3917-3920. doi:10.1021/ol0513721.

\title{
Intramolecular [4 + 2] Cycloadditions of Benzynes with Conjugated Enynes, Arenynes, and Dienes
}

\author{
Martin E. Hayes, Hiroshi Shinokubo, and Rick L. Danheiser ${ }^{*}$ \\ Department of Chemistry, Massachusetts Institute of Technology, Cambridge, Massachusetts \\ 02139
}

Abstract

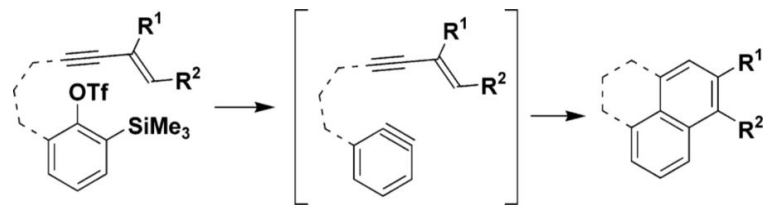

Benzynes generated by the reaction of $o$-(trimethylsilyl)aryl triflates with TBAT participate in intramolecular [4+2] cycloadditions with conjugated enynes, arenynes, and dienes to furnish highly condensed polycyclic aromatic compounds.

In this Letter we describe a new variant of the intramolecular [4 + 2] cycloaddition of conjugated enynes that provides an expeditious route to complex, highly condensed polycyclic aromatic compounds. In this transformation, conjugated enynes $1^{-3}$ (and "arenynes" 4 ) combine at room temperature with substituted benzynes5 in an unusually facile cycloaddition to generate isoaromatic cyclic allenes of general type 2 (eq 1$) .6$ These highly strained intermediates then rearrange via either proton or radical-mediated hydrogen atom transfer pathways ${ }^{1,7}$ to afford the desired polycyclic aromatic systems $(\mathbf{3})$.

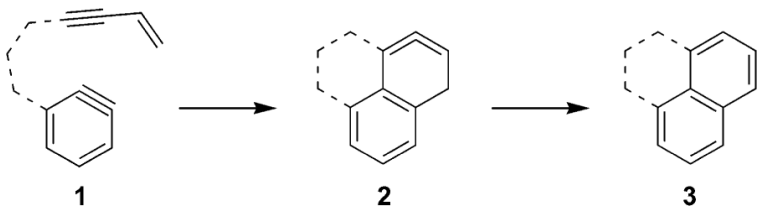

Arynes are fascinating species that engage in a variety of interesting transformations, including nucleophilic addition reactions, $[2+2]$ cycloadditions, and Diels-Alder reactions. ${ }^{5}$ DielsAlder reactions with cisoid dienes such as furan are especially well-known, though reactions involving acyclic dienes are often complicated by competing [2 +2$]$ cycloaddition and ene reaction pathways. Surprisingly, only a single report has previously appeared on the intramolecular Diels-Alder reaction of benzynes with acyclic dienes.8 In this 1995 study, Buszek obtained the desired Diels-Alder adduct in only 20-28\% yield, although a related cycloaddition with a cyclic diene (constrained to an s-cis conformation) was found to proceed

(C) 2005 American Chemical Society

*danheisr@mit.edu .

Supporting Information Available: Experimental procedures, characterization data, and ${ }^{1} \mathrm{H}$ NMR spectra for all new compounds. This material is available free of charge via the Internet at http://pubs.acs.org. 
in good yield. ${ }^{9}$ To our knowledge, the intramolecular cycloaddition of an aryne and conjugated enyne (eq 1) has not been described previously, although several examples of low-yield intermolecular cycloadditions of benzyne with aryl acetylenes have been reported. ${ }^{10} \mathrm{We}$ therefore considered it worthwhile to undertake a systematic investigation of the feasibility and scope of the intramolecular [ $4+2]$ cycloaddition of arynes with conjugated enynes and related species.

The first subgoal in this study was to identify the optimal protocol for the generation of arynes in the context of the proposed cycloaddition. Among the various methods available for generating benzynes, ${ }^{5}$ procedures based on the desilylation of ortho-substituted arenes appeared to be particularly well suited for our purposes. We chose to utilize the protocol introduced by Kobayashi involving the fluoride-induced 1,2-elimination of $o$-(trimethylsilyl) aryl triflates, which proceeds under mild conditions and employs readily available phenol derivatives as substrates. ${ }^{11}$ Scheme 1 outlines the assembly of a typical benzyne precursor. Directed metalation of the MOM ether derivative of phenol with $n$-BuLi followed by silylation furnished the expected arylsilane, which in the same flask was subjected to a second ortho lithiation followed by reaction with DMF. Careful hydrolysis ${ }^{12}$ then afforded the known $o$ silyl salicylaldehyde 5. ${ }^{13}$ Reductive amination with propargylamine and Sonogashira coupling ${ }^{14}$ with isopropenyl bromide provided enyne 7 , which was converted to the desired cycloaddition substrate by sulfonylation with excess triflic anhydride. An important consideration in the design of this strategy was the expected ability of $\mathbf{6}$ to serve as a common precursor to a variety of enynes and arenynes required in our projected systematic study of the scope of the proposed cycloaddition.

Initial attempts to effect the desired aryne cycloaddition using the Kobayashi protocol were disappointing. Although the desired reaction was observed to take place at room temperature with 1.5-2.0 equiv of TBAF in THF ( $0.005-0.01 \mathrm{M}, 1.5$ equiv of BHT), the cycloadduct 9 was obtained in only 24-32\% yield. Employing 2 equiv of $\mathrm{CsF}$ in $\mathrm{CH}_{3} \mathrm{CN}$ (1.5 equiv of BHT, rt, $46 \mathrm{~h}$ ) for benzyne generation improved the yield to $43 \%$, and reaction with 4 equiv each of $\mathrm{KF}$ and 18-crown-6 (6 equiv of BHT, THF, rt, $24 \mathrm{~h}$ ) led to the isolation of the desired product in $79 \%$ yield. ${ }^{15}$ After considerable experimentation, the optimized procedure outlined in eq 2 was developed. Most important to the success of the reaction is the use of easily handled $\operatorname{TBAT}^{16}\left(n-\mathrm{Bu}_{4} \mathrm{NSiPh}_{3} \mathrm{~F}_{2}\right)$, a commercially available and nonhygroscopic fluoride source. Best results are obtained when the reaction is conducted in a dilute $(0.005 \mathrm{M})$ solution in THF in the presence of 1.5 equiv of BHT.17,18 At higher concentrations (e.g., $0.1 \mathrm{M}$ ), the yield declines to $41 \%$, most likely due to competing intermolecular side reactions involving the reactive intermediate benzyne.

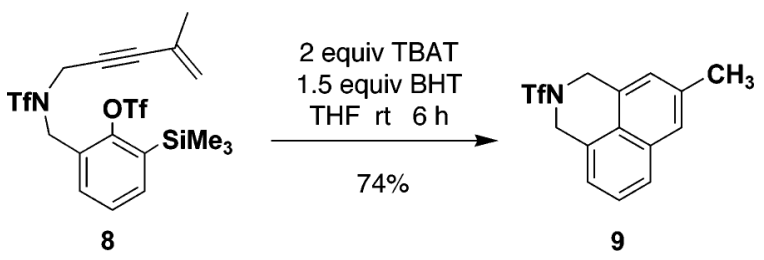

Having developed optimal conditions for effecting the desired cycloaddition in the case of enyne 8, we turned our attention to exploring the scope of the reaction with respect to substitution on the enyne moiety. Table 1 summarizes our results. Cycloaddition substrates 10-14 were all easily prepared via terminal acetylene 6 using Sonogashira coupling reactions with alkenyl halides and triflates (see Supporting Information). Most of the cycloadditions examined were found to proceed smoothly, although substrates lacking substituents at the 
internal position of the enyne double bond give the desired products in low yield (Table 1, entries 2 and 4). ${ }^{19}$ Attempts to identify byproducts from these reactions were unsuccessful, and only uncharacterizable polymers were observed by ${ }^{1} \mathrm{H}$ NMR analysis of the crude products.

Intramolecular cycloadditions also proceed in good yield in the case of related substrates in which the enyne double bond is incorporated in an aromatic or heteroaromatic ring. Table 2 presents several examples of these [4 +2] cycloadditions of benzynes with "arenyne" and "hetarenyne" $4 \pi$ components. Interestingly, attempted cycloaddition with the 3 -substituted thiophene $\mathbf{2 3}$ under our standard conditions produced a 2:1 mixture of the expected cycloadduct $\mathbf{2 7}$ and a byproduct identified as $\mathbf{2 8}$ (Figure 1) in a $45 \%$ combined yield. In this case, reaction is best performed in the absence of BHT, and under these conditions the desired cycloadduct is obtained cleanly in $51 \%$ yield after purification by column chromatography. We believe that the formation of $\mathbf{2 8}$ likely proceeds via combination of a phenoxy radical with a thiyl radical generated by $\mathrm{C}-\mathrm{S}$ homolysis of a radical intermediate formed during the isomerization of the cyclic allene involved in this reaction.

The efficiency of the cycloadditions of conjugated enynes and arenynes with benzynes generated under the conditions described here led us to investigate the intramolecular DielsAlder reaction of acyclic dienes with benzynes using a similar protocol. Scheme 2 outlines the synthesis of a Diels-Alder cycloaddition precursor via a route analogous to that employed for the preparation of our enyne substrates. In this case, reductive amination with allylamine followed by sulfonylation provided $\mathbf{2 9}$, which was subjected to cross-metathesis ${ }^{20}$ with methyl vinyl ketone and then Wittig olefination to afford the desired cycloaddition substrate 30. In contrast to the intramolecular Diels-Alder reactions of benzynes with acyclic dienes reported previously, ${ }^{8}$ cycloaddition of $\mathbf{3 0}$ under our conditions proved to be remarkably efficient. Thus, treatment of $\mathbf{3 0}$ with 2.0 equiv of TBAT at room temperature in THF provided the desired Diels-Alder adduct $\mathbf{3 1}$ in $86 \%$ yield after purification by column chromatography.

In summary, a new variant of the intramolecular [4 +2] cycloaddition of conjugated enynes and arenynes has been developed that provides access to highly condensed polycyclic aromatic compounds. Crucial to the success of these reactions is the method for the generation of the benzyne cycloaddition partners, which involves the TBAT-promoted 1,2-elimination of $o$ (trimethylsilyl)aryl triflates. In contrast to most prior enyne and arenyne cycloadditions that require elevated temperatures, these benzyne cycloadditions proceed readily at $25^{\circ} \mathrm{C}$. The first example of an efficient intramolecular Diels-Alder reaction of a benzyne with an acyclic diene has been achieved by employing similar conditions.

\section{Supplementary Material}

Refer to Web version on PubMed Central for supplementary material.

\section{Acknowledgments}

We thank the National Institutes of Health (GM 28273) for generous financial support. M.E.H. was supported in part by a predoctoral fellowship from the Ford Foundation. We thank Dr. Kazuya Matsunaga and Dr. Ralf Demuth for important exploratory experiments.

\section{References}

(1). For recent examples of thermal and Lewis-acid-promoted enyne and "heteroenyne" cycloadditions, see: (a) Danheiser RL, Gould AE, Fernandez de la Pradilla R, Helgason AL. J. Org. Chem 1994;59:5514.. (b) Wills MSB, Danheiser RL. J. Am. Chem. Soc 1998;120:9378.. (c) Dunetz JR, Danheiser RL. J. Am. Chem. Soc 2005;127:5776. [PubMed: 15839661] and references therein. 
(2). Early examples of enyne and arenyne cycloadditions are reviewed in: Onishchenko, AS. Diene Synthesis. Israel Program for Scientific Translations; Jerusalem: 1964. p. 249-254.p. 635-637..

(3). For reviews of transition-metal-catalyzed enyne cycloadditions, see: (a) Rubin M, Sromek AW, Gevorgyan V. Synlett 2003:2265.. (b) Saito S, Yamamoto Y. Chem. Rev 2000;100:2901. [PubMed: 11749309].

(4). For recent examples of cycloadditions of conjugated arenynes (the "Michael-Bucher reaction"), see: (a) Rodríguez D, Martínez-Esperón MF, Navarro-Vázquez A, Castedo L, Domínguez D, Saá C. J. Org. Chem 2004;69:3842. [PubMed: 15153017] . (b) Martínez-Esperón MF, Rodríguez D, Castedo L, Saá C. Org. Lett 2005;7:2213. [PubMed: 15901172] and references therein.

(5). Reviews: (a) Hoffmann, RW. Dehydrobenzene and Cycloalkynes. Academic Press; New York: 1967. . (b) Gilchrist, TL. The Chemistry of the Functional Groups. Patai, S.; Rappaport, Z., editors. Wiley; New York: 1983. p. 383-419.. (c) Hart, H. The Chemistry of Functional Groups. Patai, S.; Rappaport, Z., editors. Wiley; New York: 1994. p. 1017-1134.. (d) Pellissier H, Santelli M. Tetrahedron 2003;59:701..

(6). Transformation $\mathbf{1} \rightarrow \mathbf{2}$ may proceed via a concerted cycloaddition or may involve a stepwise mechanism involving an intermediate biradical. For a discussion of stepwise mechanisms in benzyne cycloadditions, see ref 5. For a discussion of biradical intermediates in arenyne cycloadditions, see: Rodríguez D, Navarro A, Castedo L, Domínguez D, Saá C. Org. Lett 2000;2:1497. [PubMed: 10841463] .

(7). For mechanistic and theoretical studies on enyne and arenyne cycloadditions, see: (a) Burrell RC, Daoust KJ, Bradley AZ, DiRico KJ, Johnson RP. J. Am. Chem. Soc 1996;118:4218.. (b) Ananikov VP. J. Phys. Org. Chem 2001;14:109.. (c) Rodríguez D, Navarro-Vázquez A, Castedo L, Domínguez D, Saá C. J. Org. Chem 2003;68:1938. [PubMed: 12608814] .

(8). Buszek KR. Tetrahedron Lett 1995;36:9125.

(9). Buszek KR, Bixby DL. Tetrahedron Lett 1995;36:9129.

(10). (a) Stiles M, Burckhardt U, Haag A. J. Org. Chem 1962;27:4715. (b) Dyke SF, Marshall AR, Watson JP. Tetrahedron 1966;22:2515. (c) Cobas A, Guitián E, Castedo L. J. Org. Chem 1997;62:4896.

(11). Himeshima Y, Sonoda T, Kobayashi H. Chem. Lett 1983:1211.For an earlier report on the generation of benzynes by fluoride-promoted elimination of $o$-(trimethylsilyl)aryl chlorides, see: Cunico RF, Dexhemier EM. J. Organomet. Chem 1973;59:153..

(12). Williams DR, Barner BA, Nishitani K, Phillips JG. J. Am. Chem. Soc 1982;104:4708.

(13). O'Connor KJ, Wey S-J, Burrows CJ. Tetrahedron Lett 1992;33:1001.

(14). For a review of alkyne cross-coupling, see: Negishi E, Anastasia L. Chem. Rev 2003;103:1979. [PubMed: 12744698] .

(15). Interestingly, Kobayashi reports that none of the expected cycload-duct with furan was obtained when benzyne generation was attempted using KF under similar conditions (ref 10a).

(16). Pilcher AS, DeShong P. J. Org. Chem 1996;61:6901. [PubMed: 11667585]

(17). Cycloadduct 9 was obtained in $54 \%$ yield when 1.5 equiv of TBAT and 0.5 equiv of BHT were employed.

(18). Under the same conditions but in the absence of BHT, the cycloadduct 9 is obtained in only $27 \%$ yield. We believe that BHT serves as a hydrogen and/or proton donor in facilitating the isomerization of cyclic allene intermediates of type $\mathbf{2}$ to the aromatic products.

(19). We speculate that substitution at this position suppresses side reactions of the intermediate cyclic allene 2, possibly including intermolecular trapping by benzyne intermediates.

(20). (a) Connon SJ, Blechert S. Angew. Chem., Int. Ed 2003;42:1900. (b) Chatterjee AK, Choi T-L, Sanders DP, Grubbs RH. J. Am. Chem. Soc 2003;125:11360. [PubMed: 16220959] 
1) $n$-BuLi, then $\mathrm{Me}_{3} \mathrm{SiCl}$

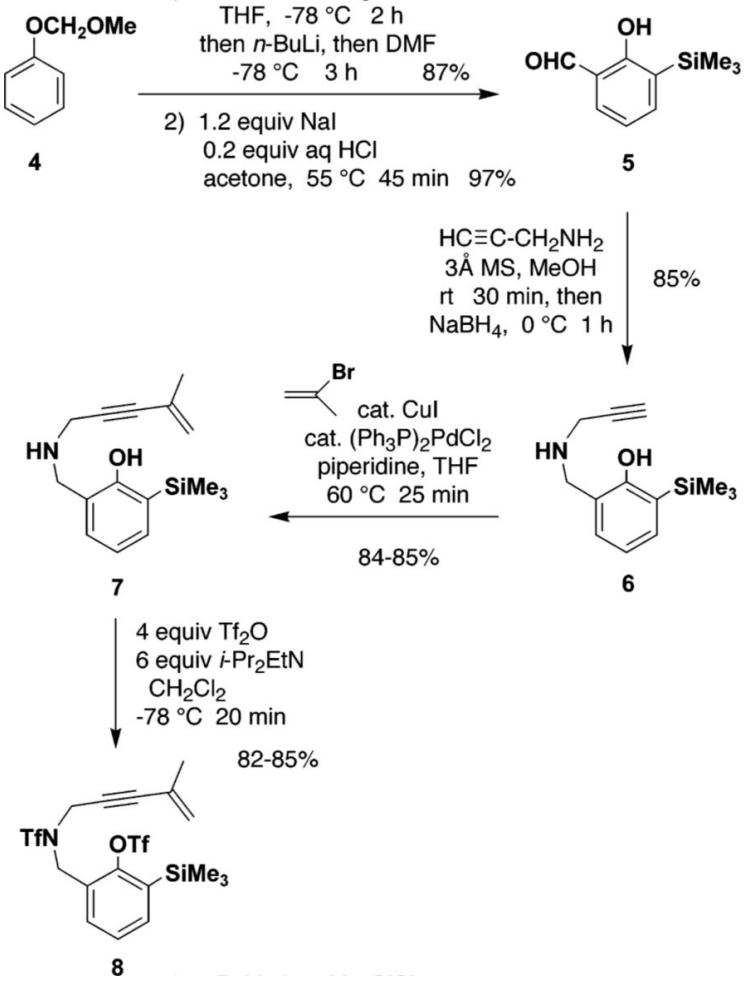

Scheme 1.

Synthesis of Typical Cycloaddition Substrate 

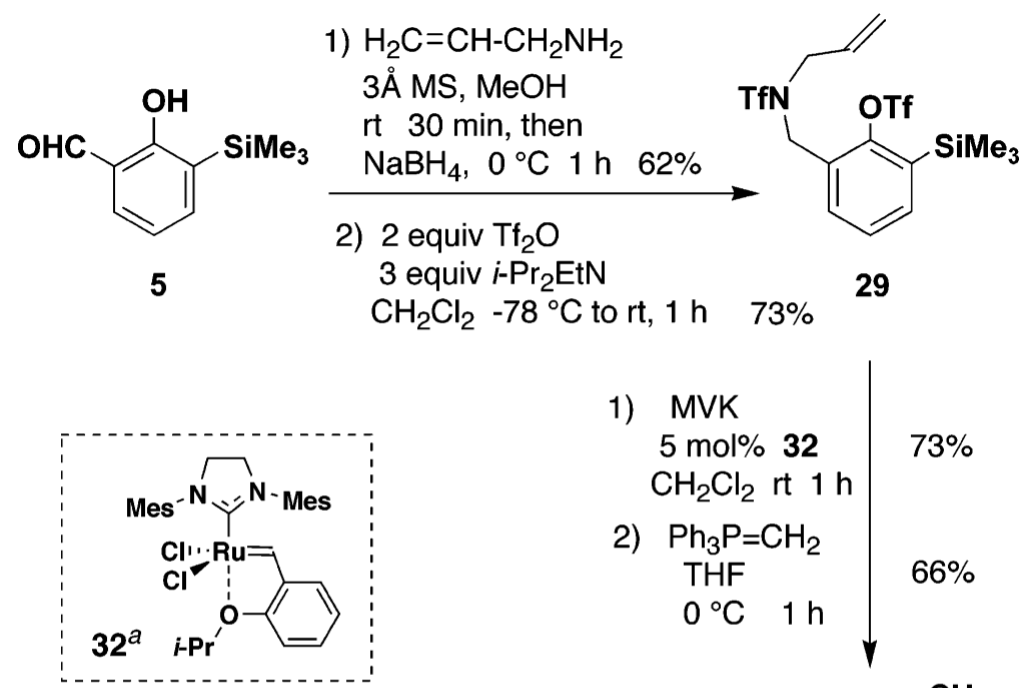

1) $\mathrm{MVK}$

$5 \mathrm{~mol} \% 32$

$73 \%$ $\mathrm{CH}_{2} \mathrm{Cl}_{2}$ it $1 \mathrm{~h}$

2) $\mathrm{Ph}_{3} \mathrm{P}=\mathrm{CH}_{2}$ THF $0{ }^{\circ} \mathrm{C} \quad 1 \mathrm{~h}$ $66 \%$

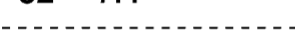

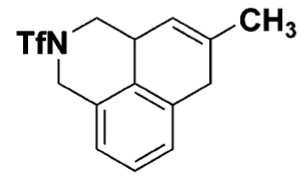

31

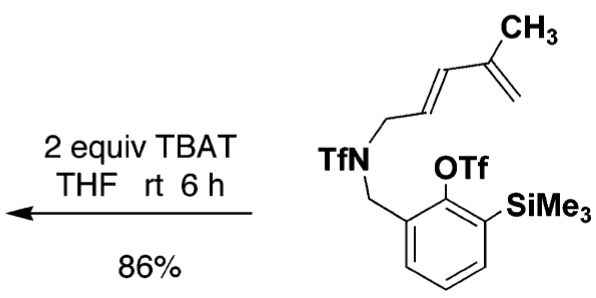

30

Scheme 2.

Study of Intramolecular Cycloaddition of a Benzyne and Acyclic Diene

${ }^{a}$ Garber, S. B.; Kingsbury, J. S.; Gray, B. L.; Hoveyda, A. H. J. Am. Chem. Soc. 2000, 122, 8168. 


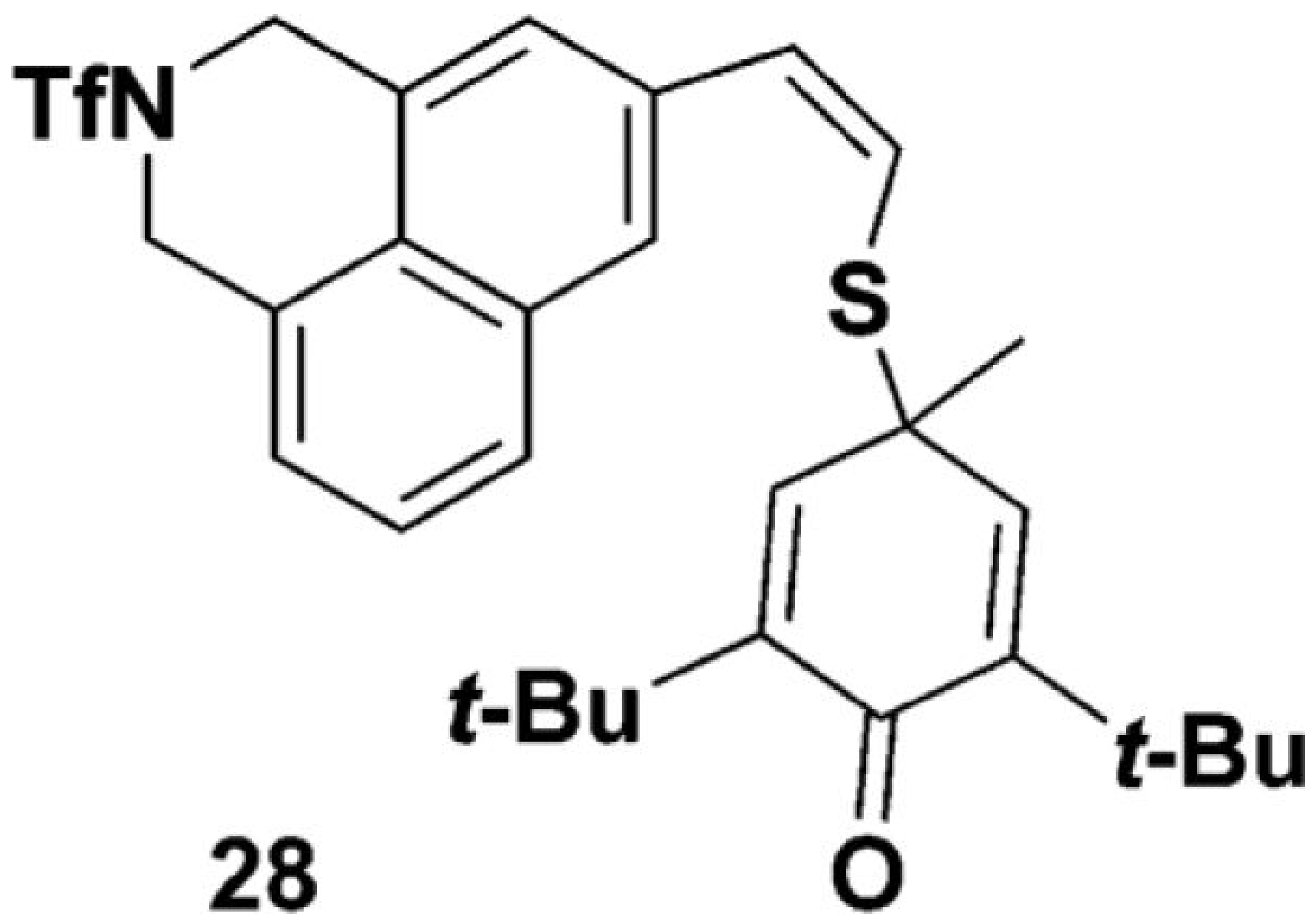

Figure 1.

Byproduct obtained in cycloaddition of $\mathbf{2 3}$ when the reaction is carried out in the presence of BHT. 
Table 1

Cycloaddition of Arynes and Conjugated Enynes 


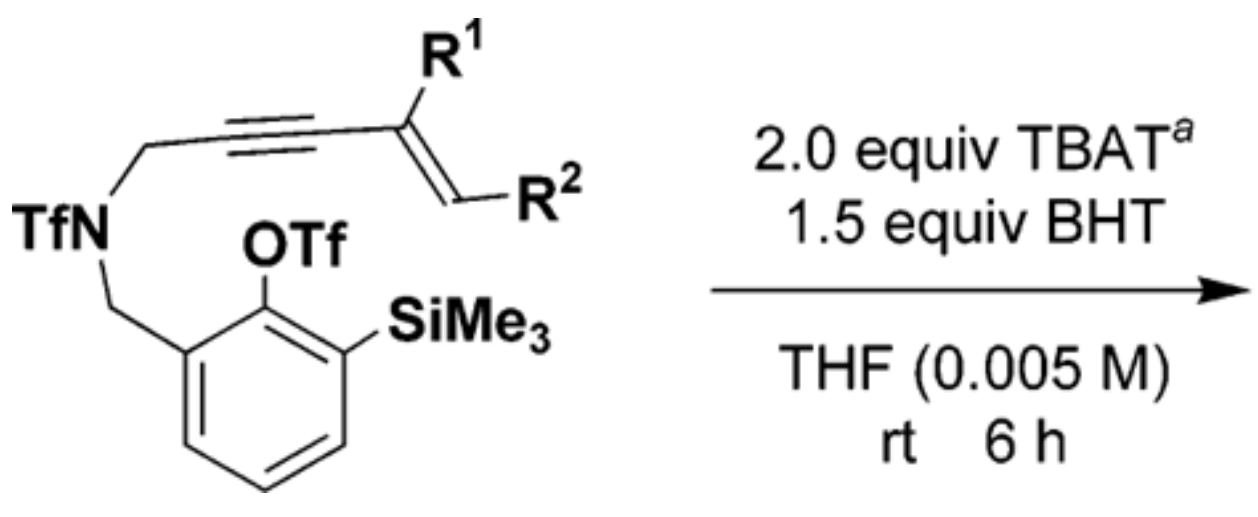

$$
\text { entry }
$$

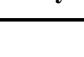

cycloaddition substrate

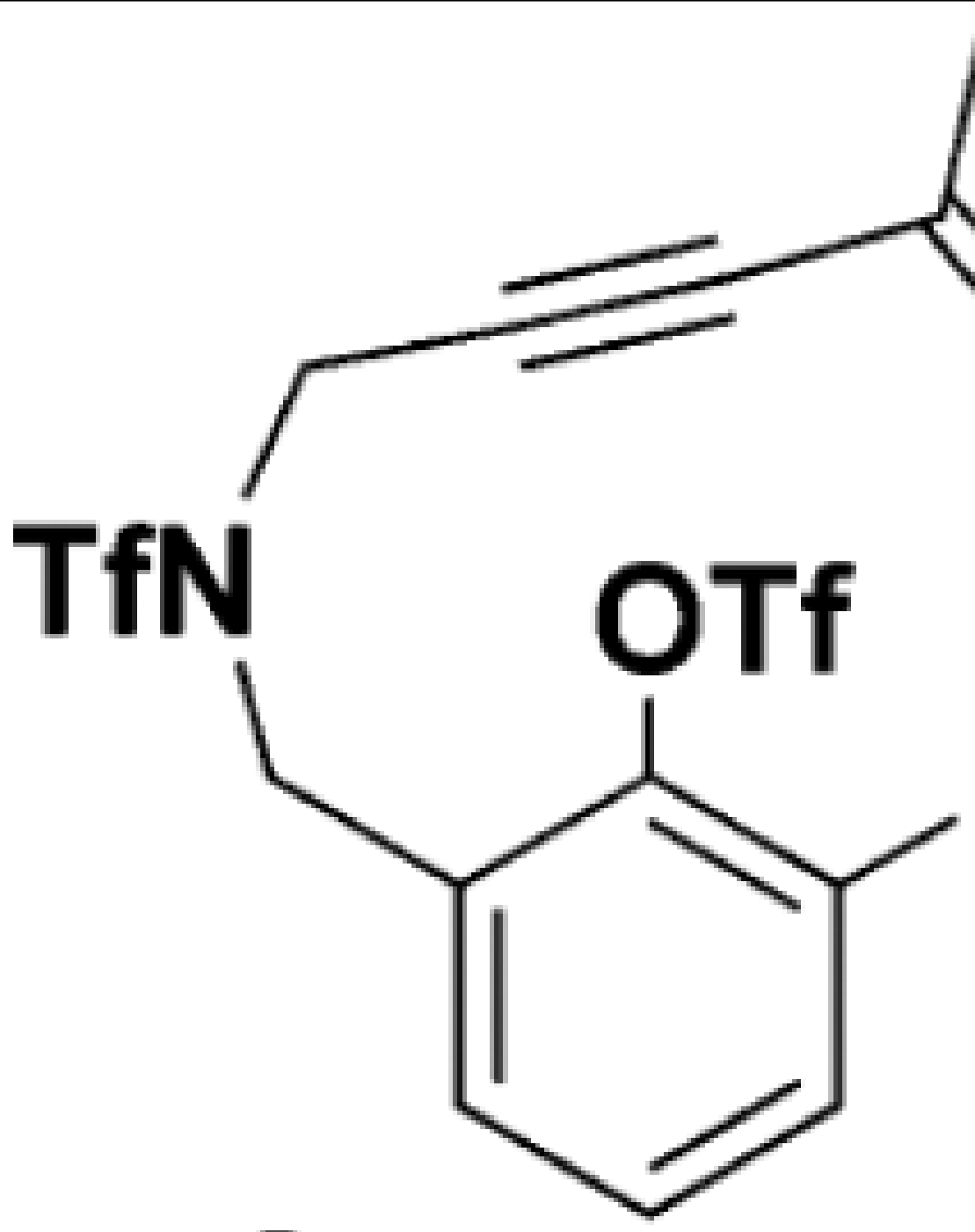

cycloaddition product
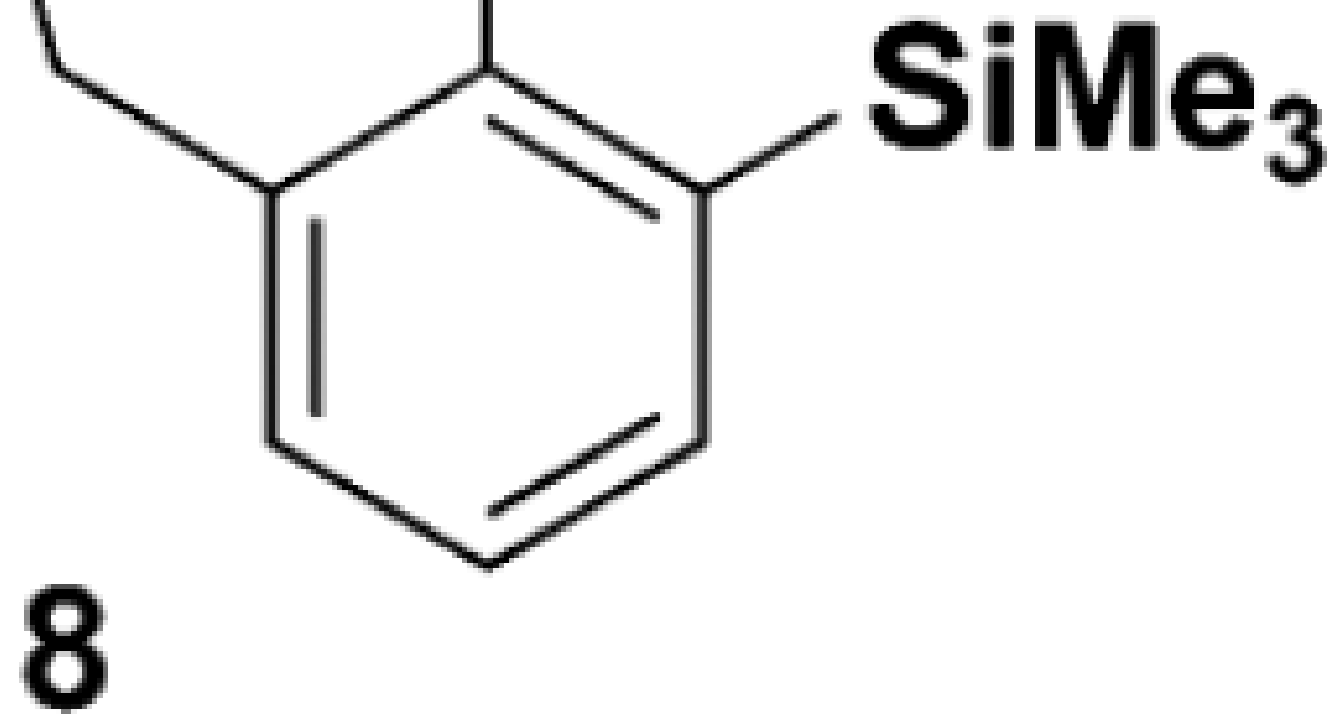

cycloadditior

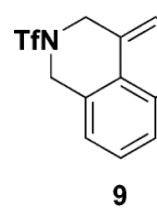

Org Lett. Author manuscript; available in PMC 2010 June 28. 

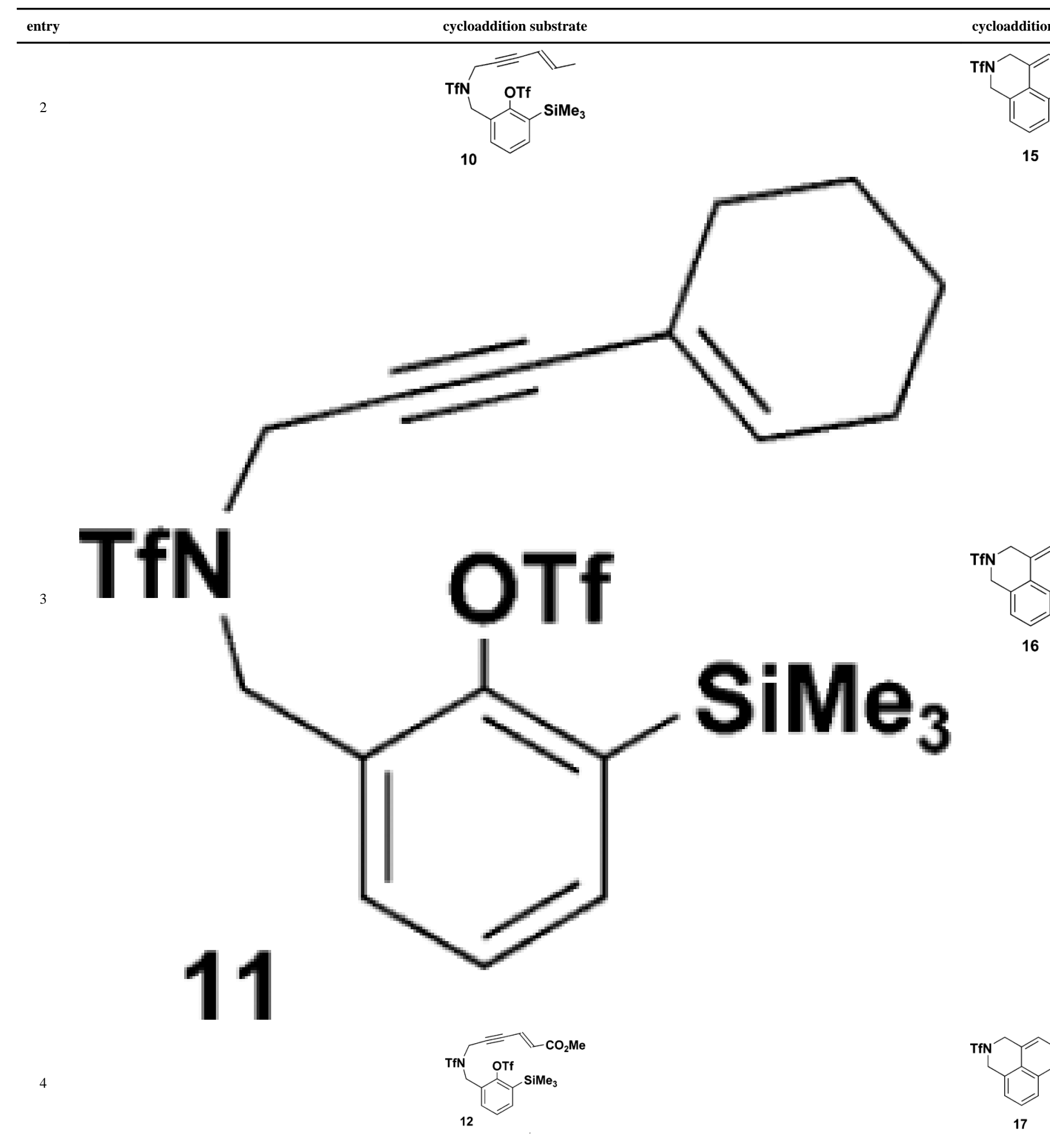

15

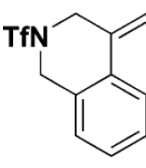

16 
entry cycloaddition substrate cycloaddition
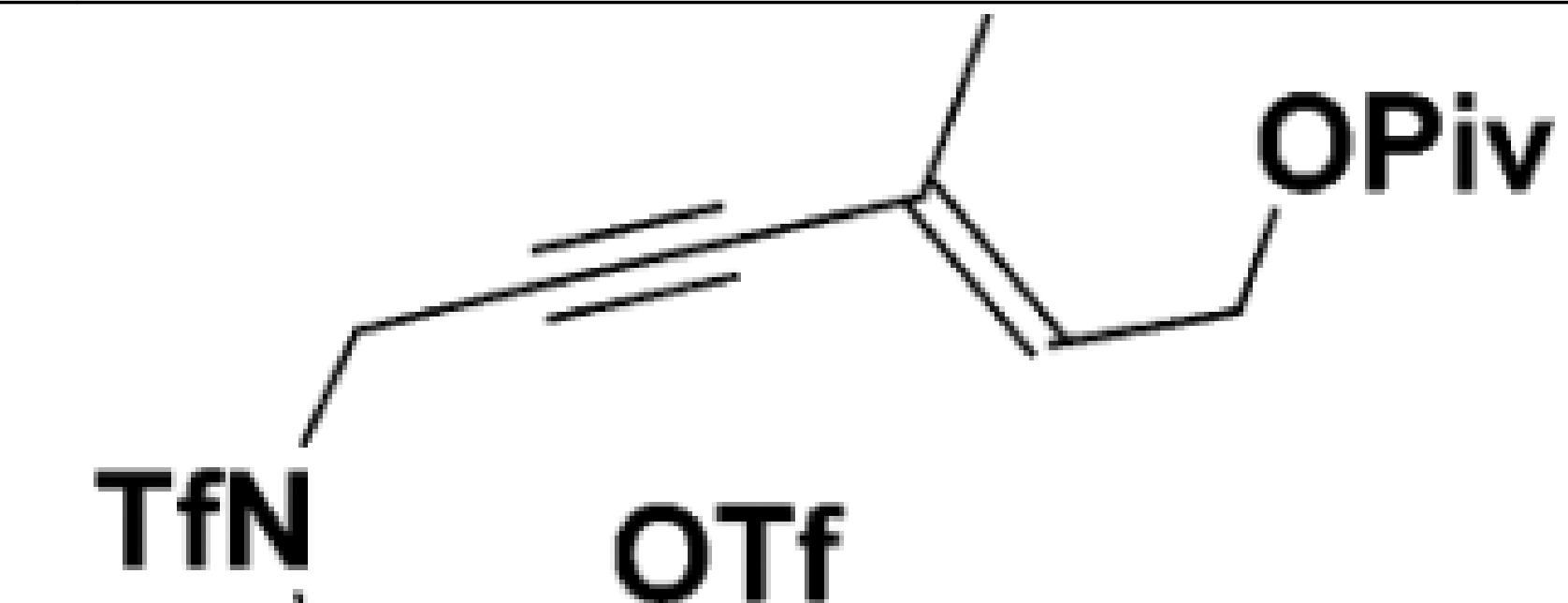

5

1

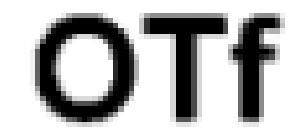

4

15
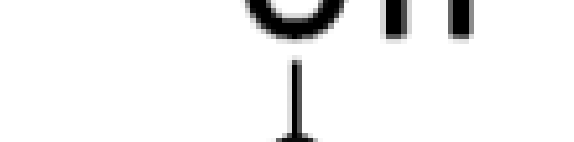

$\mathrm{SiMe}_{3}$

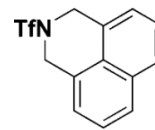

18

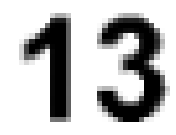

Org Lett. Author manuscript; available in PMC 2010 June 28. 


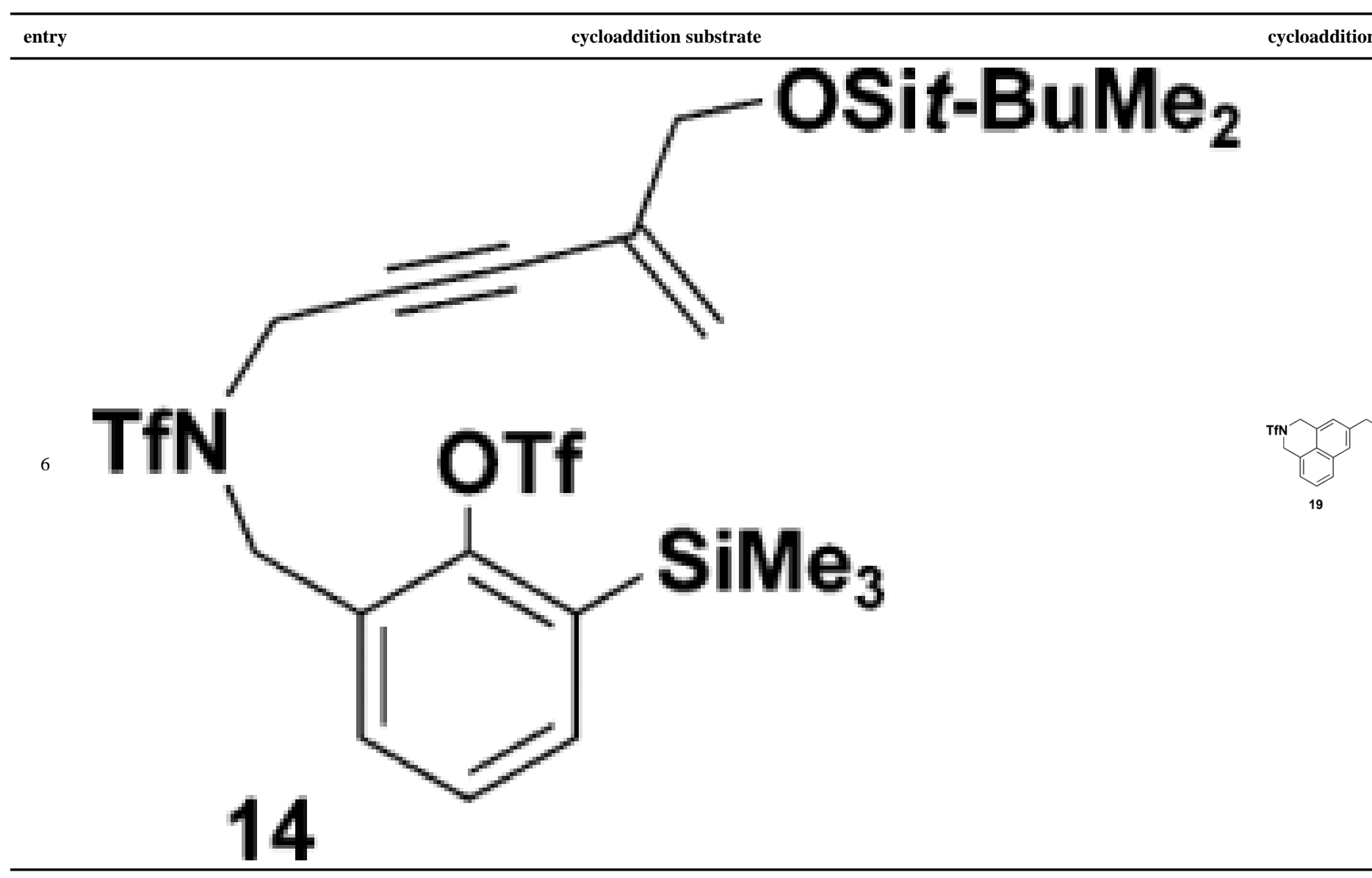

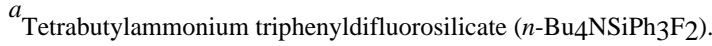

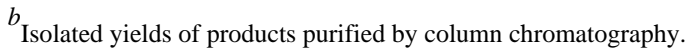


Table 2

Cycloaddition of Arynes with Arenynes and Hetarenynes

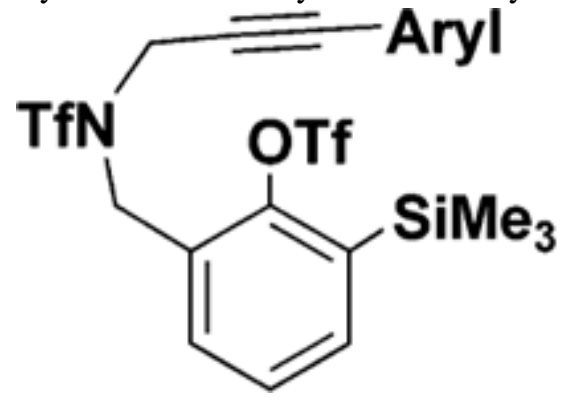

2.0 equiv TBAT $^{a}$ 1.5 equiv $\mathrm{BHT}$

entry

cycloaddition substrate

\section{THF $(0.005 \mathrm{M})$}

it $6 \mathrm{~h}$

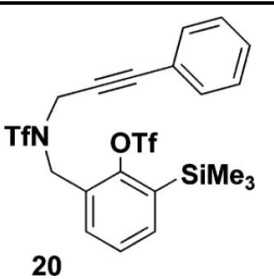


entry cycloaddition substrate
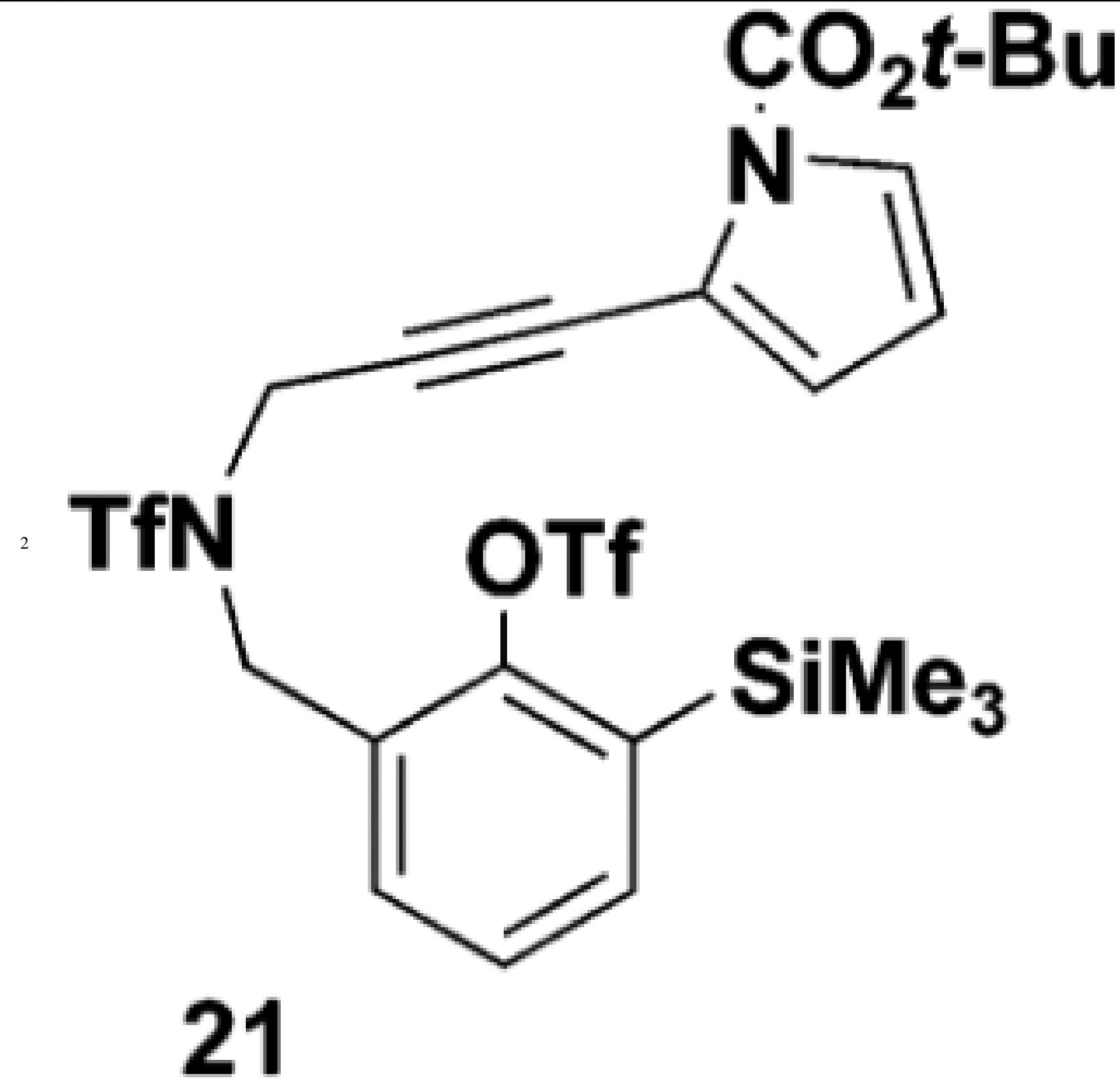

Org Lett. Author manuscript; available in PMC 2010 June 28. 
entry cycloaddition substrate

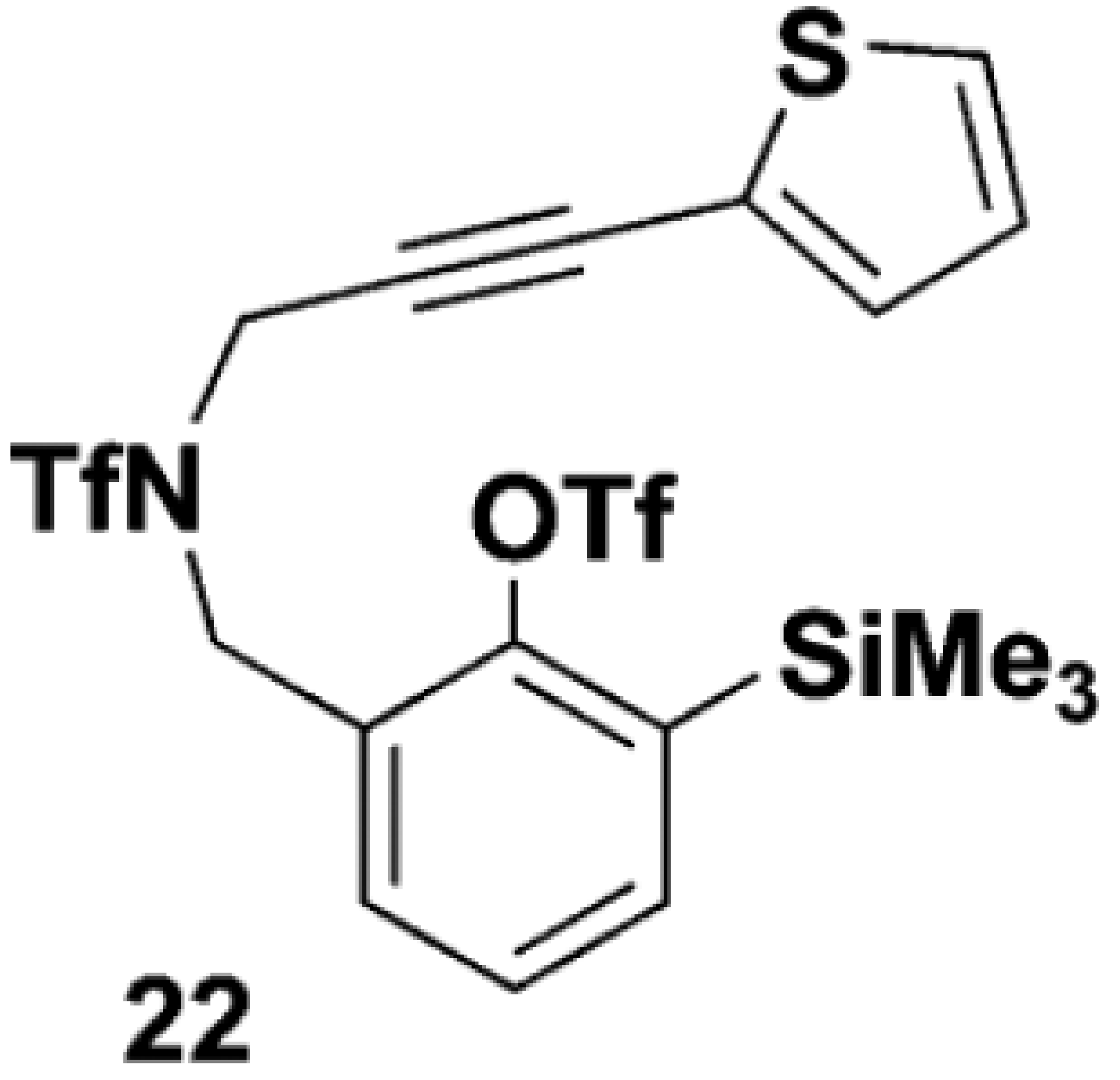




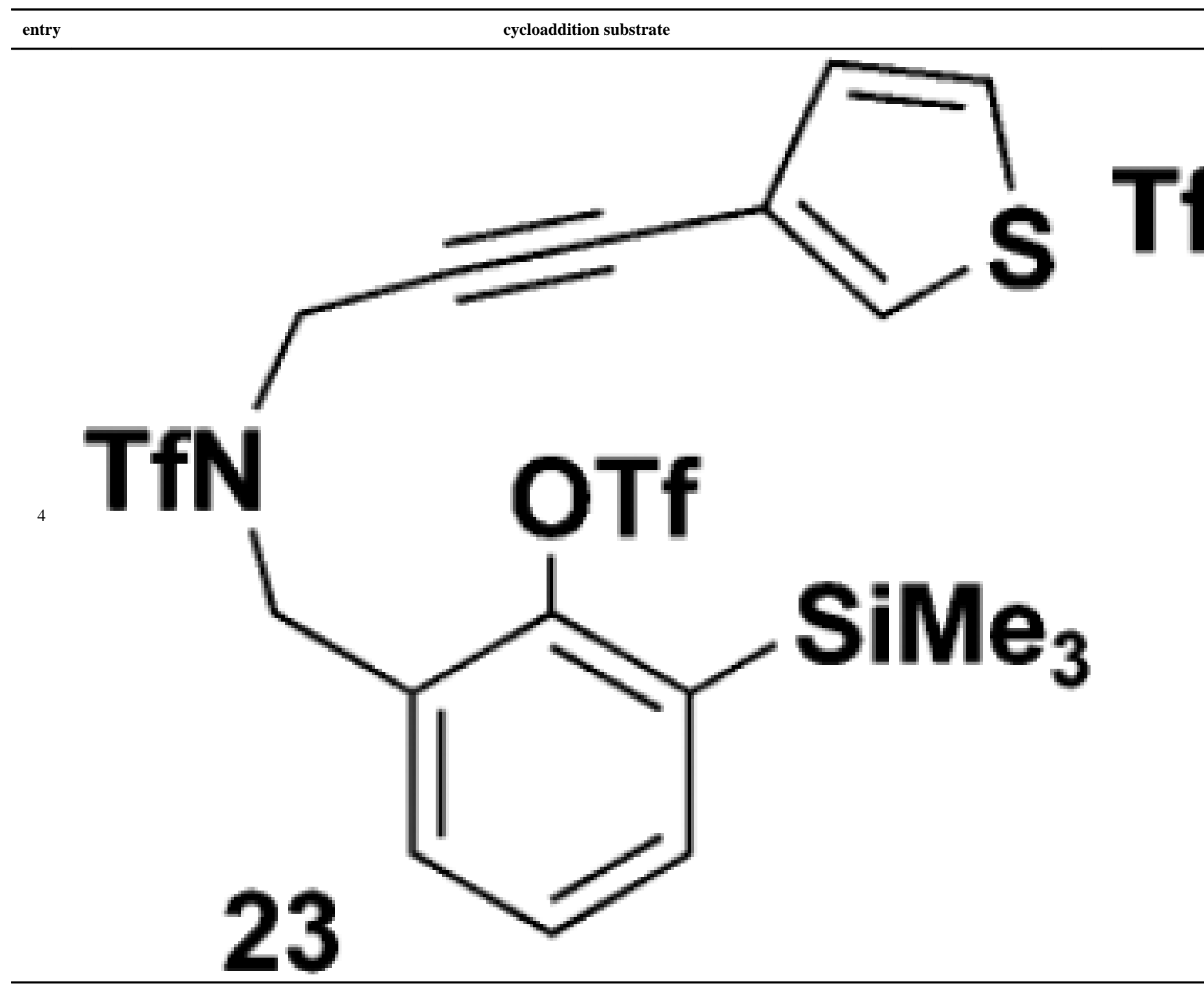

${ }^{a}$ Tetrabutylammonium triphenyldifluorosilicate $\left(n-\mathrm{Bu}_{4} \mathrm{NSiPh}_{3} \mathrm{~F}_{2}\right)$.

${ }^{b}$ Isolated yields of products purified by column chromatography.

${ }^{c}$ Reaction in the absence of BHT. 